This item was submitted to Loughborough's Research Repository by the author.

Items in Figshare are protected by copyright, with all rights reserved, unless otherwise indicated.

\title{
Using bubble functions in the multi-scale finite element modeling of the convection-diffusion-reaction equation
}

PLEASE CITE THE PUBLISHED VERSION

\section{PUBLISHER}

(C) Elsevier

\section{VERSION}

AM (Accepted Manuscript)

\section{LICENCE}

CC BY-NC-ND 4.0

\section{REPOSITORY RECORD}

Parvazinia, Mahmoud, and Vahid Nassehi. 2010. "Using Bubble Functions in the Multi-scale Finite Element Modeling of the Convection-diffusion-reaction Equation”. figshare. https://hdl.handle.net/2134/5946. 
This item was submitted to Loughborough's Institutional Repository (https://dspace.lboro.ac.uk/) by the author and is made available under the following Creative Commons Licence conditions.

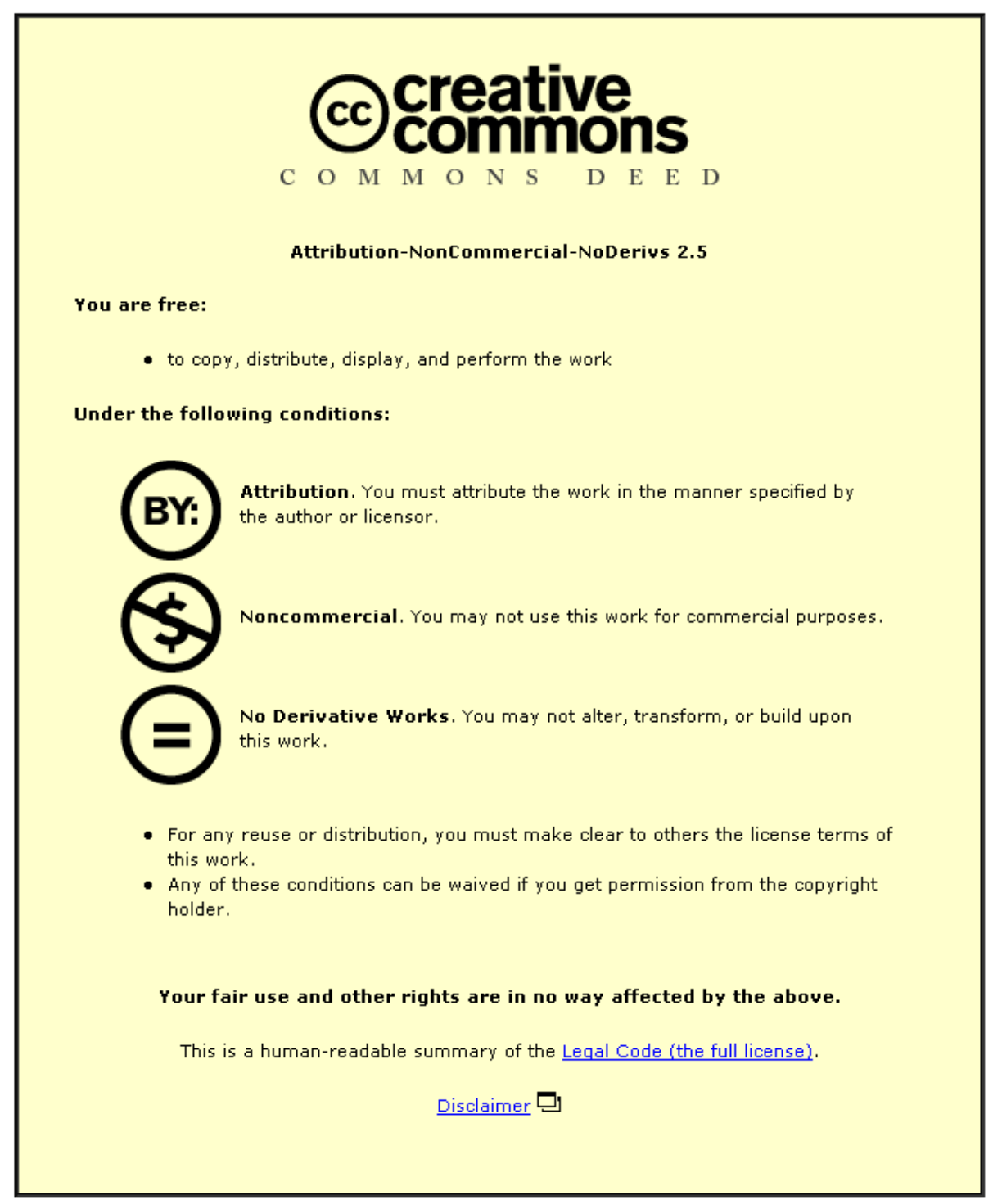

For the full text of this licence, please go to: http://creativecommons.org/licenses/by-nc-nd/2.5/ 


\title{
Using bubble functions in the multi-scale finite element modeling of the convection- diffusion-reaction equation
}

\author{
M.Parvazinia ${ }^{1}$, V.Nassehi ${ }^{2}$ \\ 1- Iran Polymer and Petrochemical Institute, P.O.Box: 14965/115, Tehran, Iran. \\ 2- Department of Chemical Engineering, Loughborough University, Loughborough, \\ Leicestershire, LE11 3TU, UK
}

\begin{abstract}
The Convection-diffusion-reaction (CDR) equation shows multi-scale behaviour in cases where it represents convection or reaction dominated transport processes. Bubble function enriched finite elements are used to generate stable and accurate solutions for this equation. Bubble functions are added to the ordinary Lagrange shape functions using either residual free bubble (RFB) functions or other simpler bubble functions in conjunction with the static condensation (STC) methods. To validate the approach, the numerical results obtained for a benchmark problem are compared with their corresponding analytical solution. Further numerical experiments are carried out to investigate the performance of the developed scheme over a range of Peclet and Damkohler numbers for productive and dissipative reaction cases.
\end{abstract}

Keywords: Convection-diffusion-reaction equation, Bubble function enriched finite elements, Multi-scale transport processes.

Corresponding author: Vahid Nassehi: V.Nassehi@lboro.ac.uk

\section{Introduction}

The general mathematical model incorporating different types of transport phenomena is expressed as the CDR equation. In general, however, the solution of this equation cannot be assumed to be globally smooth. In particular, if the field variables vary rapidly within 
thin layers adjacent to domain boundaries, or internal layers, sharp gradients are produced and standard numerical schemes lead to inaccurate and unstable results. Almost all of such situations can be regarded as multi-scale phenomena in which both fine and coarse scale variations of field variables need to be taken into account in the numerical solution of the CDR equation. Theoretically, any basically sound scheme should generate accurate numerical results if sufficiently refined computational grids are used. In practice, however, such an approach will not be computationally cost effective.

These complications can be resolved using variational multi-scale methods $[1,2]$. The multi-scale approach can be applied to situations where traditional methods can only be used in conjunction with very fine discretizations. Therefore this technique offers a general method for the modeling of transport problems with multi-scale behaviour. Amongst such problems turbulent flow, convection-diffusion processes and flow in porous media can be considered. In all of these problems, the simultaneous representation of all of the governing physical phenomena requires very high levels of mesh refinement or artificial smoothing, otherwise the fine scale information is ignored resulting in the generation of unstable and inaccurate [3] solutions. In the variational multi-scale method, the field unknown (T) is divided into two parts as $T=T_{1}+T_{b}$, where $T_{b}$ represents the fine scale variations of $T$ and may be derived analytically whilst $T_{1}$, the coarse scale variations of $T$, is approximated using standard polynomial finite element discretizations. To generate practical multi-scale schemes the bubble enhanced trial functions can be used in a finite element context. Bubble functions are, typically, high order polynomials which vanish on the element boundaries [4-7]. A systematic approach to derive bubble functions is the residual free bubble (RFB) method [8-11]. In this method, the governing differential equation is solved within each element subject to homogeneous boundary conditions.

The behavior of the CDR equation has mainly been studied under exponential regimes [12-17]. To study the CDR equation in both exponential and propagation regimes Hauke [18] has developed a sub-grid scale model based on a time-scale parameter, originally defined and formulated by Hughes [1]. 
In this paper the bubble functions are used for multi-scale finite element modeling of CDR equation in both exponential and propagation regimes for a relatively wide range of Peclet and Damkohler numbers. In multi-dimensional problems the analytical solution of the CDR equation can represent major difficulties. To overcome such problems a semidiscrete method is developed in which the solution of the PDE is replaced by the analytical solution of ordinary differential equations [19]. In this technique the exact solutions obtained from the ODE is expanded using the Taylor series and the multidimensional bubble functions are derived by tensor products of one-dimensional functions. The resulting functions are polynomial bubble functions which, for example, have been used to model the flow in porous media by Parvazinia et al. [19].

The method of incorporating bubble functions with Lagrangian shape functions using both semi-discrete and the static condensation methods are explained in the solution of the CDR equation.

\section{Governing equations}

The steady state convection-diffusion-reaction equation in domain $\Omega \subset R^{d}$ can be written as

$$
V \cdot \nabla T-k \nabla \cdot \nabla T-s T=f
$$

Where $V$ is the velocity vector, $\mathrm{k}$ is the diffusion (conduction) coefficient and $s$ is a source/sink term ( $\mathrm{s}>0$ represents production and $\mathrm{s}<0$ stands for dissipation), $T$ is the field unknown, $f$ is a given source term. Using the following dimensionless forms

$$
\left\{\begin{array}{l}
T^{*}=\frac{T}{T_{1}} \\
\bar{x}^{*}=\frac{\bar{x}}{h}
\end{array}\right.
$$

where superscript * represents the dimensionless variables, $T_{1}$ is a reference value of the field variable, $h$ is a characteristic length ( e.g. width of the domain) and $\bar{x}$ represents 
position vector in the selected coordinate system. After substitution from equation (2) the general governing equation is written in a dimensionless form as

$\nabla T^{*}-\frac{1}{P_{e}} \nabla \cdot \nabla T^{*}-D_{a} T^{*}=f^{*}$

in which $f^{*}$ is the dimensionless source term, $P_{e}$ is the Peclet number and $D_{a}$ is the Damkohler number, respectively, defined as

$$
\left\{\begin{array}{l}
P_{e}=\frac{v h}{k} \\
D_{a}=\frac{s h}{v} \\
f^{*}=\frac{h}{T_{1} v} f
\end{array}\right.
$$

It is assumed that $P_{e}$ is equal in both directions. A similar assumption is made for $D_{a}$ (see Figs.1 and 6). In a two-dimensional system $\left(x^{*}, y^{*}\right)$ Eq. (3) can be written as

$$
\left(\frac{\partial T^{*}}{\partial x^{*}}+\frac{\partial T^{*}}{\partial y^{*}}\right)-\frac{1}{P_{e}}\left(\frac{\partial^{2} T^{*}}{\partial x^{* 2}}+\frac{\partial^{2} T^{*}}{\partial y^{* 2}}\right)-D_{a} T^{*}=f^{*}
$$

Corresponding dimensionless boundary conditions for the rectangular domain are:

\section{a) Dissipation (see Fig.1):}

$$
\begin{aligned}
& T^{*}=0 \quad \text { for } y^{*}=0, \quad 0 \leq x^{*} \leq 1 \text { and } x^{*}=0,0 \leq y^{*}<1 \\
& T^{*}=1 \quad \text { for } \quad x^{*}=1, \quad 0 \leq y^{*}<1 \text { and } y^{*}=1,0 \leq x^{*} \leq 1
\end{aligned}
$$

b) Production (see Fig.6):

$$
\begin{array}{ll}
T^{*}=0 & \text { for } x^{*}=0, \quad 0 \leq y^{*} \leq 1 \\
T^{*}=1 & \text { for } x^{*}=1, \quad 0 \leq y^{*} \leq 1 \\
\frac{\partial T^{*}}{\partial x^{*}}=\frac{\partial T^{*}}{\partial y^{*}}=0 & \text { for } \quad y^{*}=0 \text { and } y^{*}=1 \text { at } 0<x^{*}<1
\end{array}
$$

\section{Standard Galerkin finite element scheme}


After discretization of the solution domain into a computational mesh, consisting of predetermined geometrical shapes, the prime unknowns in the governing equations are replaced by approximate forms defined within the selected finite elements. In the weighted residual finite element scheme, used in the present work, these unknowns are replaced by trial function representations, which in the context of a discretized domain are given by low order interpolation polynomials, $N_{j}[20]$ as

$T^{*} \approx \tilde{T}^{*}=\sum_{j=1}^{n} N_{j} T_{j}^{*}$

where $n$ is total number of nodes in an element and, $T_{j}^{*}$ is the nodal values of unknown at the nodes (i.e. sampling points) of an element. Therefore, the above equation provides approximate values for unknown within an element via interpolation using its nodal values. Substitution of approximate values for the unknown from Eq. (8) into the governing Eq. (5), leads to the appearance of residual statements. These statements are then multiplied by appropriate weight functions $\left(W_{i}\right)$ and integrated over an element domain. In the standard Galerkin method, the selected weight functions are identical to the interpolation functions $\left(W_{i}=N_{i}\right)$. In the multi-scale scheme using bubble functions the interpolation functions are enriched with bubble function and the weight and interpolation functions are not equal. . Following the described steps we obtain

$\int_{\Omega_{e}}\left[W_{i}\left(\frac{\partial \sum_{j=1}^{n} N_{j} T_{j}^{*}}{\partial x^{*}}+\frac{\partial \sum_{j=1}^{n} N_{j} T_{j}^{*}}{\partial y^{*}}\right)-\frac{1}{P_{e}} W_{i}\left(\frac{\partial^{2} \sum_{j=1}^{n} N_{j} T_{j}^{*}}{\partial x^{* 2}}+\frac{\partial^{2} \sum_{j=1}^{n} N_{j} T_{j}^{*}}{\partial y^{* 2}}\right)-D_{a} W_{i} \sum_{j=1}^{n} N_{j} T_{j}^{*}-W_{i} f^{\circ}\right] d x d y=0$

The second order differentials in Eq. (9) are reduced by the application of Green's theorem (i.e. generalised form of integration by parts). This leads to the appearance of boundary integral (flux) terms along the exterior boundaries of finite elements. For each interpolation function an identical weight function can be used to generate weighted residual equations such as Eq. (9). Therefore corresponding to a total of $n$ interpolation 
functions, $n$ equations are generated and a system of $n \times n$ equations is constructed. Using matrix notation this system is written as [21]:

$$
\left[A_{i j}\right]\left\{T_{j}^{*}\right\}=\left\{B_{j}\right\}
$$

where

$$
\begin{aligned}
& A_{i j}=\int_{\Omega_{e}}\left[W_{i}\left(\frac{\partial N_{j}}{\partial x^{*}}+\frac{\partial N_{j}}{\partial y^{*}}\right)+\frac{1}{P_{e}}\left(\frac{\partial W_{i}}{\partial x^{*}} \frac{\partial N_{j}}{\partial x^{*}}+\frac{\partial W_{i}}{\partial y^{*}} \frac{\partial N_{j}}{\partial y^{*}}\right)-D_{a} W_{i} N_{j}\right] d x^{*} d y^{*} \\
& B_{j}=\int_{\Omega_{e}} W_{i} f^{\circ} d x^{*} d y^{*}+\int_{\Gamma_{e}} W_{i}\left(\frac{\partial T^{* e}}{\partial x^{*}} n_{x}+\frac{\partial T^{* e}}{\partial y^{*}} n_{y}\right) d \Gamma_{e}
\end{aligned}
$$

Using isoparametric mapping of elements from the global mesh into a master element, where all of the calculations are carried out, the uniformity of the matrix Eq. (10) can be preserved [20]. In addition, a natural coordinate system such as $-1 \leq \xi, \eta \leq+1$ can be used within the master element to enable the evaluation of all integrals within its domain by Gauss quadrature method [22].

\section{Bubble function method}

Two types of bubble functions are used. A polynomial bubble function based on RFB method and a bubble function which is incorporated with the Galerkin scheme using STC method.

\subsection{Residual free bubble functions}

The derivation of the bubble functions is based on the analytical solution of the model differential equation within each element using homogeneous boundary conditions. More specifically, to derive the appropriate bubble function for the present convectiondiffusion-reaction equation we follow the method described by Franca and Russo [10] and Brezzi et al. [23]. In a simple word the procedure consist of solving the differential equation within the element subjected to the element boundary conditions.

For the CDR equation with respect to Eq. (3), operator $L$ is defined as

$$
L=\nabla-\frac{1}{P_{e}} \Delta-D_{a}
$$


For a linear element on each node we have

$$
\begin{aligned}
& \left\{\begin{array}{l}
-\frac{1}{P_{e}} \frac{d^{2} N_{1}}{d x^{2}}+\frac{d N_{1}}{d x}-D_{a} N_{1}=0 \quad \text { for } x \in[0-l] \\
N_{1}=\psi_{1} \Rightarrow\left\{\begin{array}{l}
N_{1}(0)=1 \\
N_{1}(l)=0
\end{array}\right.
\end{array}\right. \\
& \left\{\begin{array}{l}
-\frac{1}{P_{e}} \frac{d^{2} N_{2}}{d x^{2}}+\frac{d N_{2}}{d x}-D_{a} N_{2}=0 \\
N_{2}=\psi_{2} \Rightarrow\left\{\begin{array}{l}
N_{2}(0)=0 \\
N_{2}(l)=1
\end{array}\right.
\end{array}\right.
\end{aligned}
$$

Where $l$ is the characteristic element length and $\psi_{i}$ is a linear shape function. The solution of the above equation gives bubble shape functions expressed in a local elemental coordinate system as

$$
\left\{\begin{array}{l}
N_{1}=\frac{\exp \left(\left(0.5 P_{e}-\alpha\right) l+\left(0.5 P_{e}+\alpha\right) x\right)-\exp \left(\left(0.5 P_{e}+\alpha\right) l+\left(0.5 P_{e}-\alpha\right) x\right)}{\exp \left(0.5 P_{e}-\alpha\right) l-\exp \left(0.5 P_{e}+\alpha\right) l} \\
N_{2}=\frac{\exp \left(0.5 P_{e}-\alpha\right) x-\exp \left(0.5 P_{e}+\alpha\right) x}{\exp \left(0.5 P_{e}-\alpha\right) l-\exp \left(0.5 P_{e}+\alpha\right) l}
\end{array}\right.
$$

where

$$
\alpha=0.5 \sqrt{P_{e}^{2}-4 P_{e} D_{a}}
$$

More detail of residual free method can be found in [19].

\subsection{Polynomial bubble functions}

Exponential functions (14) can only be directly used if the integrals in the elemental equations are evaluated manually. However, this results in loss of flexibility and it is desirable to convert them into polynomials to make it possible to use quadrature methods in a finite element program. For derivation of polynomial bubble functions, the Taylor expansion of the exponential function is used. 
$N_{2}$ can be expanded as

$$
N_{2}=\frac{a_{1} x+a_{2} x^{2}+a_{3} x^{3}+\ldots}{a_{1} l+a_{2} l^{2}+a_{3} l^{3}+\ldots}=\frac{\left(A_{1}-A_{2}\right) x+\frac{1}{2 !}\left(A_{1}^{2}-A_{2}^{2}\right) x^{2}+\frac{1}{3 !}\left(A_{1}^{3}-A_{2}^{3}\right) x^{3}+\ldots}{\left(A_{1}-A_{2}\right) l+\frac{1}{2 !}\left(A_{1}^{2}-A_{2}^{2}\right) l^{2}+\frac{1}{3 !}\left(A_{1}^{3}-A_{2}^{3}\right) l^{3}+\ldots}
$$

Where $l$ is a characteristic element length and

$$
\begin{aligned}
& A_{1}=0.5 P_{e}+\alpha \\
& A_{2}=0.5 P_{e}-\alpha \\
& \alpha=0.5 \sqrt{P_{e}^{2}-4 P_{e} D_{a}}
\end{aligned}
$$

If the above equation is expanded based on the term $x(l-x)$ which is the minimum order bubble, the Galerkin shape functions enriched with bubble functions can be derived. For example $2^{\text {nd }}$ order is

$$
\begin{aligned}
& N_{2}=\frac{x}{l}-\frac{a_{2}}{\left(a_{1}+a_{2} l\right) l} x(l-x) \\
& a_{1}=A_{1}-A_{2} \text { and } a_{2}=\frac{1}{2 !}\left(A_{1}^{2}-A_{2}^{2}\right)
\end{aligned}
$$

$3^{\text {rd }}$ order is:

$$
\begin{aligned}
& N_{2}=\frac{x}{l}-\frac{a_{2}+a_{3} l}{\left(a_{1}+a_{2} l+a_{3} l^{2}\right) l} x(l-x)-\frac{a_{3}}{\left(a_{1}+a_{2} l+a_{3} l^{2}\right) l} x x(l-x) \\
& a_{1}=A_{1}-A_{2}, a_{2}=\frac{1}{2 !}\left(A_{1}^{2}-A_{2}^{2}\right) \text { and } a_{3}=\frac{1}{3 !}\left(A_{1}^{3}-A_{2}^{3}\right)
\end{aligned}
$$

Derivation of $N_{1}$ is not as simple as $N_{2} .2^{\text {nd }}$ order is as follows

$$
\begin{aligned}
& N_{1}=\frac{l-x}{l}+\frac{a_{2}}{\left(a_{1}+a_{2} l\right) l} x(l-x) \\
& a_{1}=A_{1}-A_{2} \text { and } a_{2}=\frac{1}{2 !}\left(A_{1}^{2}-A_{2}^{2}\right)
\end{aligned}
$$


$3^{\text {rd }}$ order can be written as

$$
\begin{aligned}
& N_{1}=\frac{a_{1}(l-x)+a_{2}\left(l^{2}-x^{2}\right)+a_{3} x(l-x)+a_{4}\left(l^{3}-x^{3}\right)}{\left(a_{1}+a_{2} l+a_{4} l^{2}\right) l} \\
& N_{1}=\frac{l-x}{l}+\frac{a_{2}+a_{3}+2 l a_{4}}{\left(a_{1}+a_{2} l+a_{4} l^{2}\right) l} x(l-x)-\frac{a_{4}}{\left(a_{1}+a_{2} l+a_{4} l^{2}\right) l}(l-x) x(l-x) \\
& a_{1}=A_{1}-A_{2}, a_{2}=\frac{1}{2 !}\left(A_{1}^{2}-A_{2}^{2}\right), a_{3}=\frac{3}{3 !}\left(A_{2} A_{1}^{2}-A_{1} A_{2}^{2}\right) \text { and } a_{4}=\frac{1}{3 !}\left(A_{1}^{3}-A_{2}^{3}\right)
\end{aligned}
$$

Similarly higher order bubble functions can be derived.

The bubble modified shape functions in the local coordinate system $\xi(-1,+1)$ cab easily

are obtained by considering that $x=\frac{l}{2}(1+\xi)$. Two dimensional shape functions and implementation in the Galerkin finite element scheme is clearly described in Parvazinia et al. [19].

\subsection{Static Condensation}

Theoretically, any function which is zero at element boundaries can be regarded as a bubble function. Therefore alternative bubble functions other than those described in the previous section can be used. In this case the bubble coefficient can be calculated by the use of STC method [24]. A simple high order bubble function can be written as

$\phi_{b}=\left(1-\xi^{2 n}\right) \quad n=1,2,3,4 \ldots$

If this bubble function is used the enriched linear Lagrangian shape functions can be written as

$$
\begin{aligned}
& N_{1}=\frac{1}{2}(1-\xi)+b_{1}\left(1-\xi^{2 n}\right) \\
& N_{2}=\frac{1}{2}(1+\xi)+b_{2}\left(1-\xi^{2 n}\right)
\end{aligned}
$$

Using static condensation method $b_{1}$ and $b_{2}$ are calculated as 


$$
\begin{aligned}
& b_{1}=\left[\frac{l}{2} D_{a}\left(1-\frac{1}{2 n+1}\right)-\left(\frac{1}{2 n+1}-1\right)\right]\left(\frac{1}{\beta}\right) \\
& b_{2}=\left[\frac{l}{2} D_{a}\left(1-\frac{1}{2 n+1}\right)+\left(\frac{1}{2 n+1}-1\right)\right]\left(\frac{1}{\beta}\right) \\
& \beta=\frac{16 n^{2}}{l P_{e}} \frac{1}{4 n-1}-\frac{l}{2} D_{a}\left(\frac{2}{2 n+1}-\frac{2}{4 n^{2}+1}\right)
\end{aligned}
$$

where $l$ is a characteristic element length. Two dimensional shape functions and implementation in the Galerkin finite element scheme are previously described in Nassehi et al. [25] and are not repeated here.

\subsection{Elimination of the boundary integrals}

In discretizations involving bubble functions inter element boundary integrals are not automatically eliminated during the assembly of elemental equations. This problem does not become apparent in the one dimensional case as the boundary integrals are reduced to simple nodal flux terms. It is shown in the work done in [19] that the bubble function does not affect the Laplacian term and therefore no boundary integral due to the bubble function exists.

\section{Analytical solution}

To validate the numerical solutions, the analytical solution of the dimensionless convection-diffusion equation is presented. The solution for the exponential regime is based on the method of separation of variables. Prescribing the boundary conditions shown in Fig. 1 the analytical solution of the CDR equation is:

$$
\begin{aligned}
& T^{*}\left(x^{*}, y^{*}\right)=\exp \left(p_{e}\left(\frac{x^{*}+y^{*}}{2}\right)\right) \sum_{n=1}^{\infty} \beta_{n} \frac{1}{1-e^{-2 \alpha}}\left\{\sin \left(n \pi x^{*}\right)\left(e^{-\frac{\alpha}{2}\left(1-y^{*}\right)}-e^{-\frac{\alpha}{2}\left(1+y^{*}\right)}\right)+\right. \\
& \sin \left(n \pi y^{*}\right)\left(e^{\frac{-\alpha}{2}\left(1-y^{*}\right)}-e^{\frac{-\alpha}{2}\left(1+y^{*}\right)}\right\}
\end{aligned}
$$

Where 


$$
\begin{aligned}
& \alpha=\sqrt{P_{e}^{2}+4 n^{2} \pi^{2}-4 D_{a} P_{e}} \\
& \beta_{n}=\frac{8 n \pi\left\{1-(-1)^{n} \exp \left(\frac{-p_{e}}{2}\right)\right\}}{p_{e}^{2}+4 n^{2} \pi^{2}}
\end{aligned}
$$

Using the boundary conditions given in Fig.6, a one dimensional solution can also be obtained for propagation regime.

$$
T^{*}\left(x^{*}\right)=\frac{\exp \left(0.5 P_{e} x^{*}\right) \sin \left(\sqrt{\left|P_{e}^{2}-4 P_{e} D_{a}\right|} x^{*}\right)}{\exp \left(0.5 P_{e}\right) \sin \left(\sqrt{\left|P_{e}^{2}-4 P_{e} D_{a}\right|}\right)}
$$

\section{Results and discussion}

The main objective of the present work has been the construction of a multi-scale finite element scheme for the general class of CDR problems. To validate the scheme its results are initially compared with the analytical solutions obtained for a bench mark problem. This comparison shows the ability of the scheme to generate theoretically expected simulations. In all figures, the polynomial bubble functions based on the residual free method are indicated by RFB and those which are incorporated with Galerkin scheme using static condensation method are shown by STC. It is assumed that the source term $\left(f^{*}\right)$ is set to be zero.

Figs. 2 to 5 show the results for the exponential regime using a 10X10 refinement. The results are presented at mid-height cross section for different values of $P_{e}$ and $D_{a}$. Fig. 2 shows the results at $P_{e}=10$ and $D_{a}=-60$ for $2^{\text {nd }}, 4^{\text {th }}$ and $6^{\text {th }}$ order RFB bubble functions. The instability in the form of undershoot emerges. Increasing the order of the bubble function eliminates undershoot to achieve stable solution. Fig. 3 represents the results at $P_{e}=50$ and $D_{a}=-20$. As the analytical solution shows by increasing the $P_{e}$ the length of the boundary layer decreases and the instability in the Galerkin solution increases. Meanwhile, the $2^{\text {nd }}$ and $4^{\text {th }}$ order bubble functions fail to yield stable solution whilst the $6^{\text {th }}$ order bubble function remains stable. This behaviour shows that by reducing the $D_{a}$ or 
increasing the $P_{e}$, where the Galerkin solution becomes unstable and the system shows stronger multi-scale behaviour, higher order bubble functions can clearly serve a more stable solution. Fig. 4 shows the results at $P_{e}=100$ and $D_{a}=-10$. By increasing the $P_{e}$ to 100 instability in the Galerkin method increases and oscillations spread over the entire domain. The $6^{\text {th }}$ order bubble is quite stable but it shows $5 \%$ undershoot which indicates strong multi-scale behaviour at $P e=100$. It is obvious from the results that increasing $P_{e}$ or reducing $D_{a}$, since the multi-scale behaviour is amplified, the stable and accurate solution can be achieved using higher order bubble functions. Fig.5 shows a comparison between RFB and STC bubble functions at $P e=50$ and $D a=-10$

Figs. 7 to 9 show the results for the propagation regime using 10X10 mesh refinements. Domain and boundary conditions are shown in Fig 6. Since in the propagation regime the multiscale behaviour is repeated in the interlyers over the entire domain therefore higher order bubble functions perform better. Thus the $8^{\text {th }}$ order bubble functions are studied. What is clear in the results the STC bubble functions in the propagation case matches better the analytical soluton. By increasing the Peclet number Galerkin method shows stronger oscillation over the domain as a result of stronger multiscale behaviour.

\section{Conclusions}

The bubble function method is used for multi-scale finite element modeling of the CDR equation. Two types of bubble functions are evaluated at different Peclet and Damkohler numbers. These results show that the polynomial bubble functions derived by the RFB method is capable of generating stable solutions for the CDR equation in the exponential regime. For propagation regime the STC method in conjunction with the bubble functions represented in Eq (21) performs better results.

In propagation regime the multi-scale behaviour is repeated over the entire domain and in comparison with exponential regime, where multi-scale behaviour is limited to the near wall boundary layer, obtaining stable-accurate solution is more difficult comparing with exponential regime and higher order bubble functions are required. 


\section{References}

[1] T.J.R Hughes, Multi scale phenomena, Green’s functions, the Dirichlet - to-Neumann formulation, subgrid scale models, bubbles and the origins of stabilized methods, Computer methods in applied mechanics and engineering 127 (1-4) (1995) 381401.

[2] T.J.R. Hughes, J.Stewart, A space time formulation for multiscale phenomena, Journal of computational and applied mathematics 74 (1-2) (1996) 217-229.

[3] M.Parvazinia, V.Nassehi, R.J.Wakeman, M.H.R. Ghoreishy, Finite element modeling of flow through a porous medium between two parallel plates using the Brinkman equation, Transport in porous media 63 (2006) 71-90.

[4] F.Brezzi, M.Bristeau, L.P.Franca, M.Mallet, G.Roge, A relationship between stablized finite element methods and the Galerkin method with bubble functions. Computer methods in applied mechanics and engineering 96 (1) (1992) 117-129.

[5] C.Baiocchi, F.Brezzi, L.P.Franca, Virtual bubbles and Galerkin-least-squares type methods, Computer methods in applied mechanics and engineering 105 (1) (1993) 125-141.

[6.] L.P.Franca, T.J.R.Hughes, R.Stenberg, Stabilized finite element methods for the Stokes problem. In: Nicolaides, R.A. and Gunzberger, M.D. (Eds.), Incompressible Fluid Dynamics-Trends and Advances, Cambridge University Press, Cambridge (1993).

[7] F.Brezzi, L.P.Franca, T.J.R. Hughes, A.Russo, $b=\int g$, Computer methods in applied mechanics and engineering 145 (3-4) (1997) 392-339.

[8] L.P.Franca, C. Farhat, A.P. Macedo, M.Lesoinne, Residual free bubbles for Helemholtz equation, International Journal for numerical methods in engineering 40 (21) (1997) 4003-4009.

[9] L.P.Franca, A.Russo, Deriving upwinding, mass lumping and selective reduced integration by residual free bubbles, Applied mathematics letters 9 (5) (1996) 8388. 
[10] L.P.Franca, A.Russo, Unlocking with residual-free bubbles, Computer methods in applied mechanics and engineering 142 (3-4) (1997) 361-364.

[11] L.P.Franca, A.Russo, Mass lumping emanating from residual free bubbles, Computer methods in applied mechanics and engineering 142 (3-4) (1997) 353-360.

[12] C.Johnson, U.Navert, J.pitkarenta, Finite element methods for linear hyperbolic problems, Computer methods in applied mechanics and engineering 45 (1-3) (1984) 285-312.

[13] T.E.Tezduyar, Y.J.Park, Discontinuity- capturing finite element formulations for nonlinear CDR equations, Computer methods in applied mechanics and engineering 59 (3) (1986) 307-325.

[14] R.Codina, Comparison of some finite element methods for solving the diffusionconvection-reaction equation, Computer methods in applied mechanics and engineering 156 (1-4) (1998) 185-210.

[15] T.W.H. Sheu, An implicit scheme for solving the convection-diffusion-reaction equation in two dimensions, Journal of computational physics 164 (1) (2000) 123142.

[16] E.Burman, B.Hansbo, Edge stabilization for Galerkin approximations of convectiondiffusion-reaction problems, Computer methods in applied mechanics and engineering 193 (15-16) (2004) 1437-1453.

[17] J.Hoffman, C. Johnson, S.Bertoluzza, Subgrid modeling of convection-diffusionreaction in one space dimension using a Haar multiresolusion analysis, Computer methods in applied mechanics and engineering 194 (1-3) (2005) 19-44.

[18] G.Hauke, A simple subgrid scale stabilized method for the advection-diffusionreaction equation, Computer methods in applied mechanics and engineering 191 (27-28) (2002) 2925-2947.

[19] M.Parvazinia, V. Nassehi, R.J.Wakeman, Multi-scale finite element modeling of laminar steady flow through highly permeable porous media, Chemical engineering science 61 (2) (2006) 586-596.

[20] O.C.Zienkiewicz, R.L.Taylor, The finite element method, McGraw-Hill, London 1994. 
[21] V. Nassehi, Practical aspects of finite element modeling of polymer processing, John Wiely \& Sons, Chichester 2002.

[22] C.F.Gerald, P.O.Wheatley, Applied numerical analysis, Addison-Wesley 1984.

[23] F.Brezzi, L.P. Franca, A.Russo, Further considerations on residual-free bubbles for advective-diffusive equations, Computer methods in applied mechanics and engineering 166 (1-2) (1998) 25-33.

[24] K.J. Bathe, Finite Element Procedures, Prentice Hall, Englewood Cliffs, N.J. 1996.

[25] V. Nassehi, M. Parvazinia, A.Khan , Multiscale finite element modeling of flow through porous media with curve and contracting boundaries, Communications in computational physics 2 (4) (2007) 723-745. 


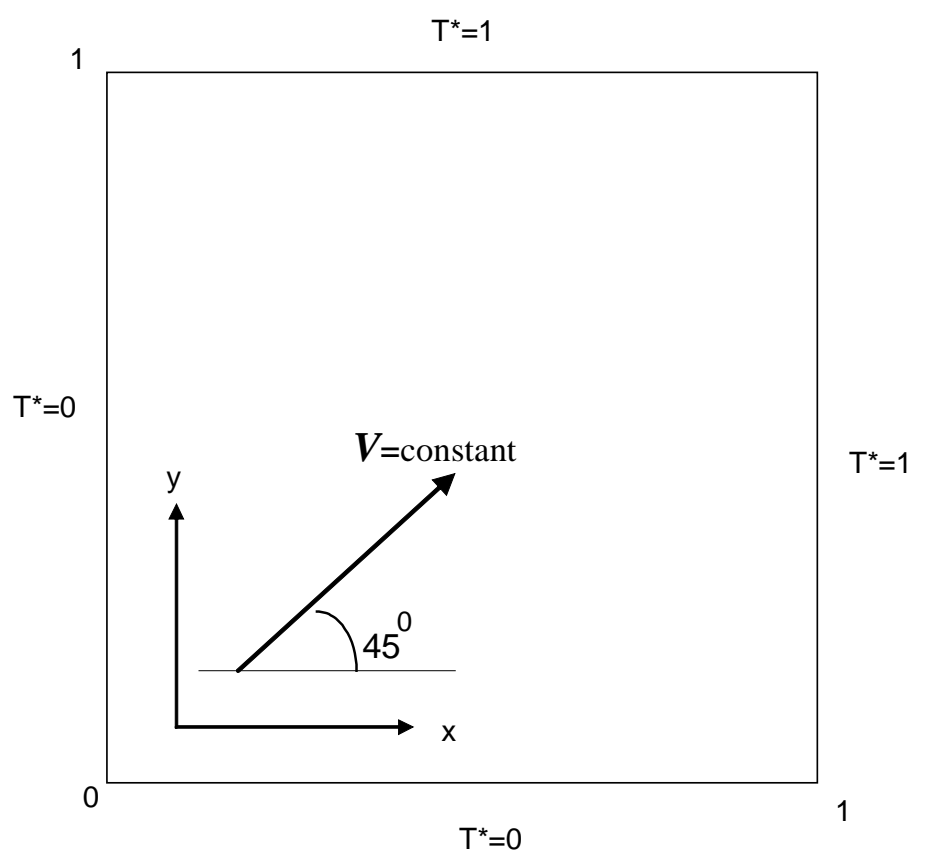

Fig. 1. Domain and the boundary conditions for the exponential regime.

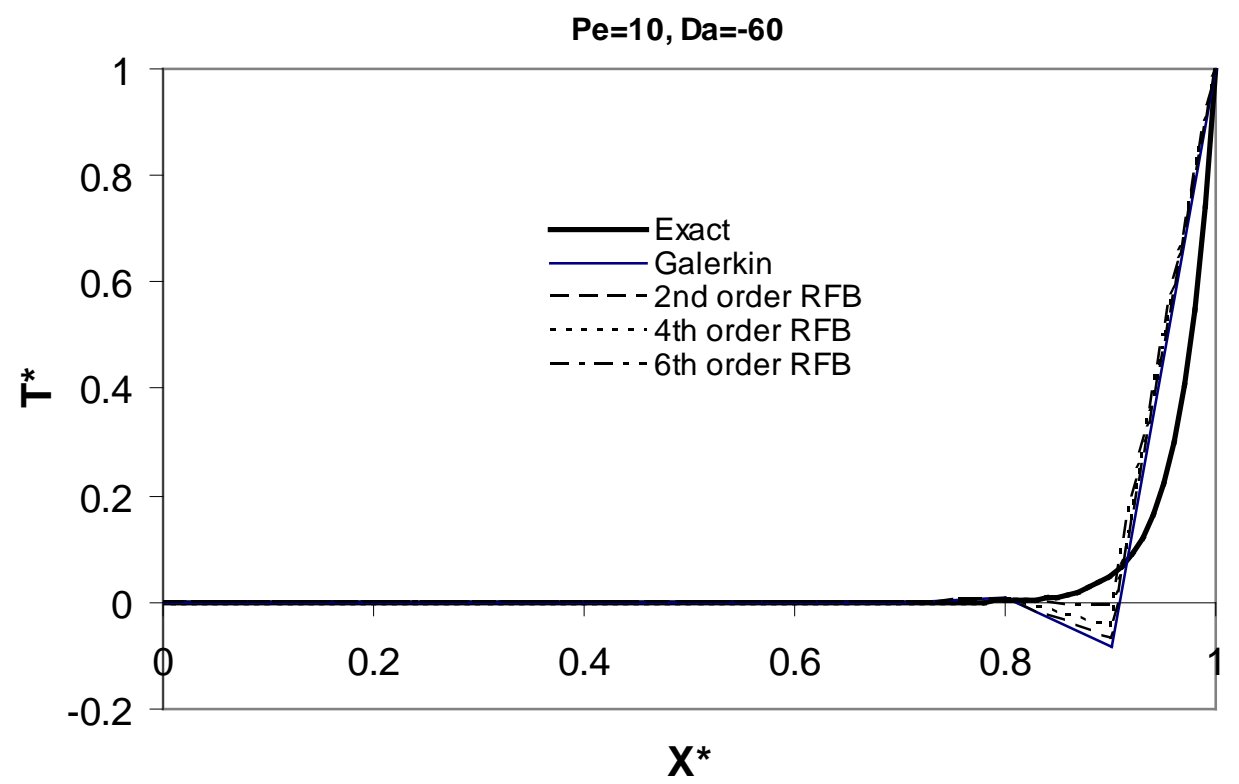

Fig. 2. Results for $P e=10$ and $D a=-60$ at $y^{*}=0.5$ - exponential regime. 


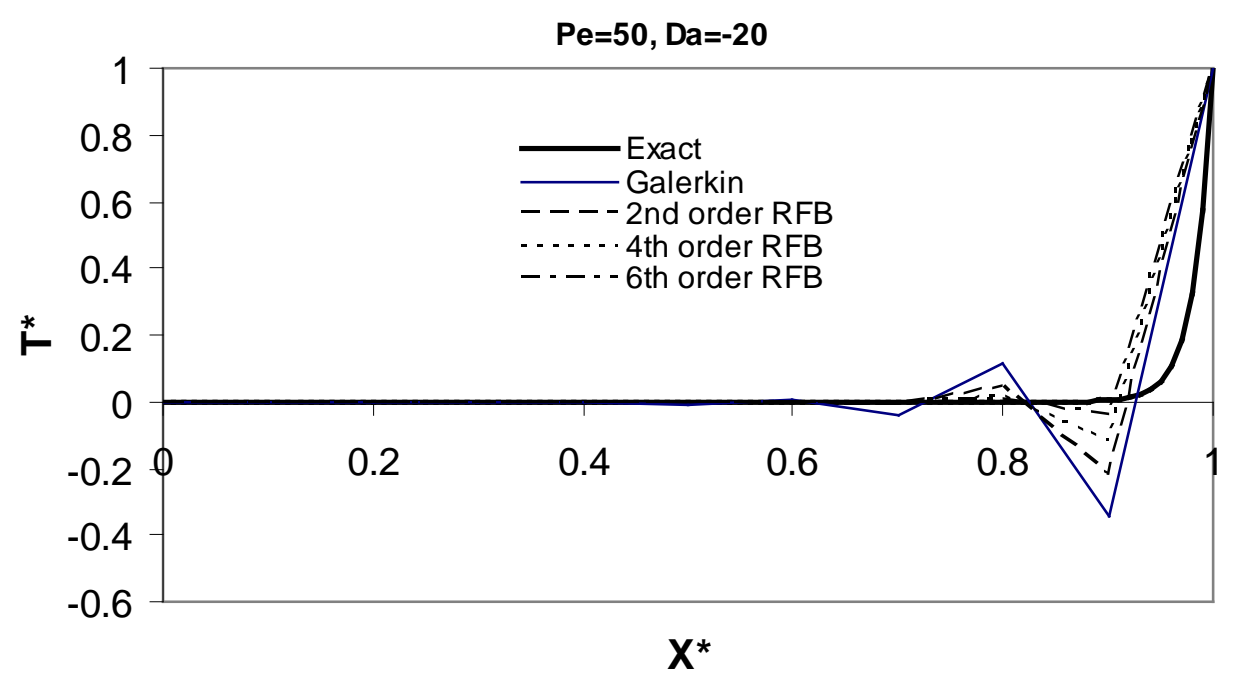

Fig. 3. Results for $P e=50$ and $D a=-20$ at $y^{*}=0.5$ - exponential regime.

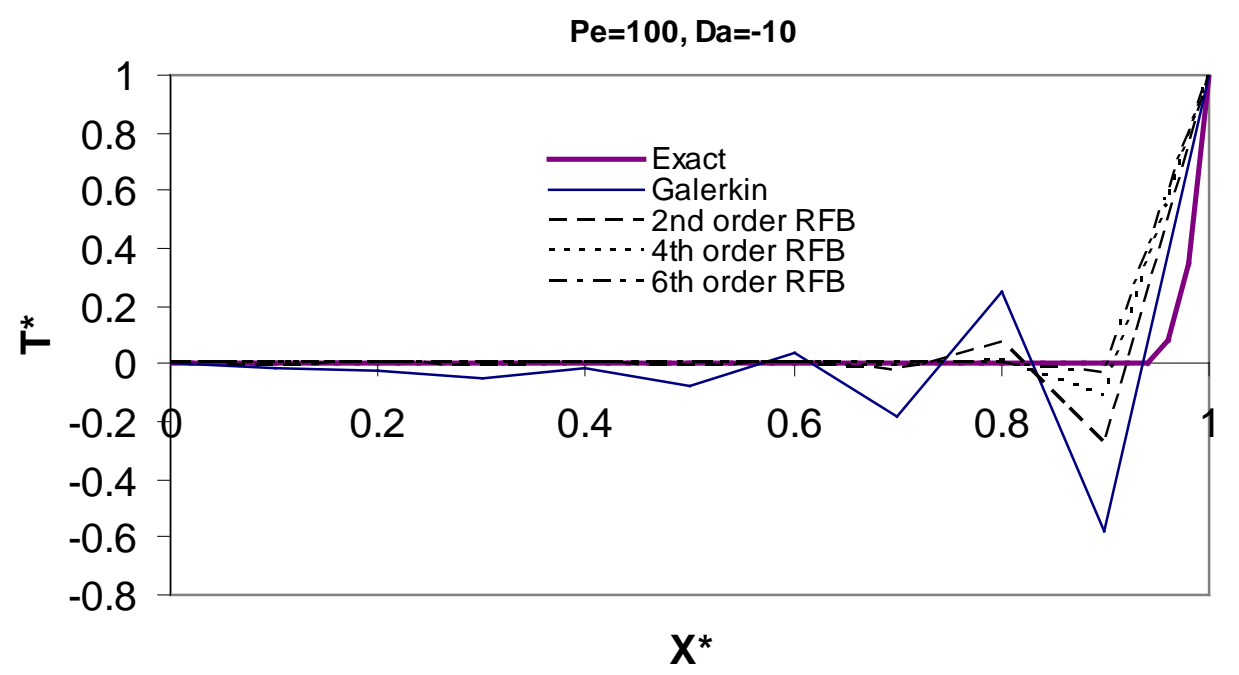

Fig. 4. Results for $P e=100$ and $D a=-10$ at $y^{*}=0.5$ - exponential regime. 


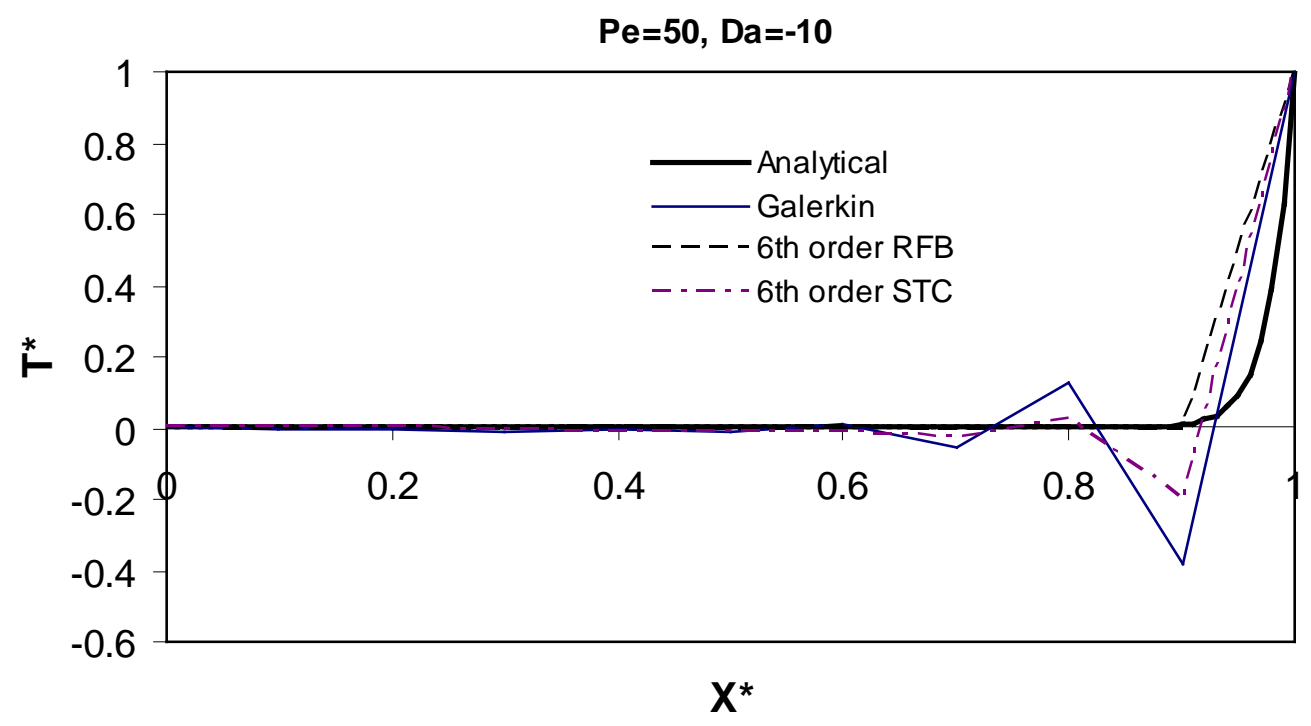

Fig. 5. Results for $P e=50$ and $D a=-10$ at $y^{*}=0.5$ - exponential regime.

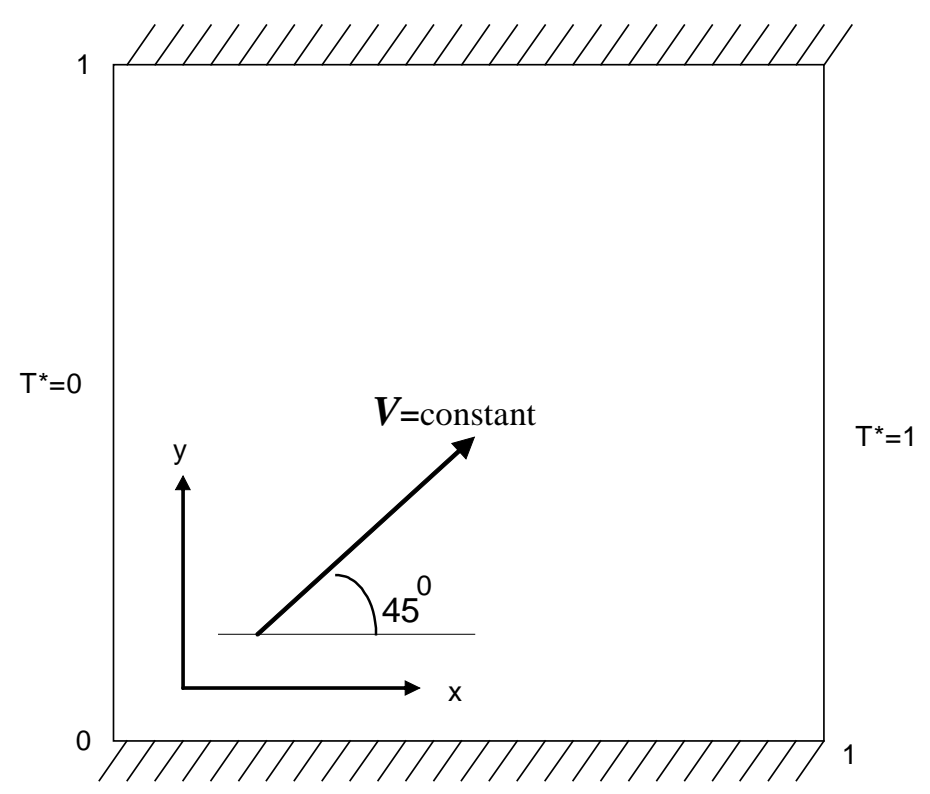

Fig. 6. Domain and the boundary conditions for the propagation regime. 


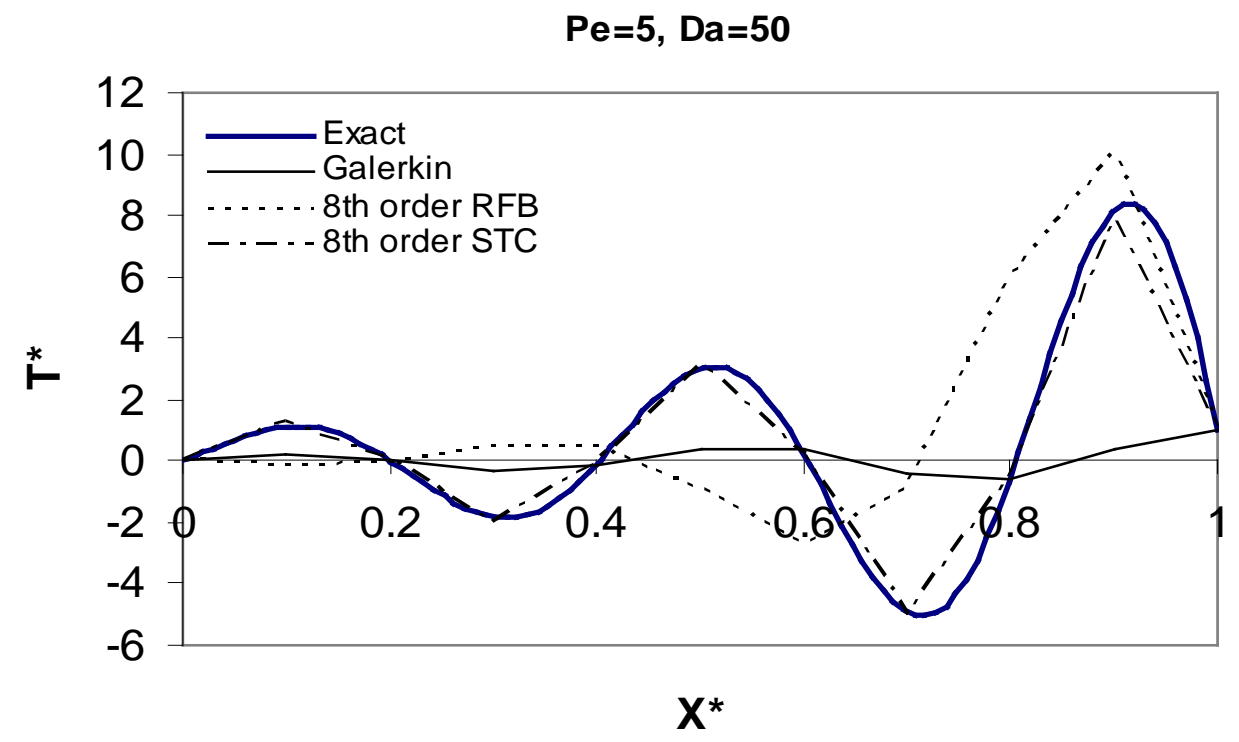

Fig. 7. Results for $P e=5$ and $D a=35,50,65$ - propagation regime.

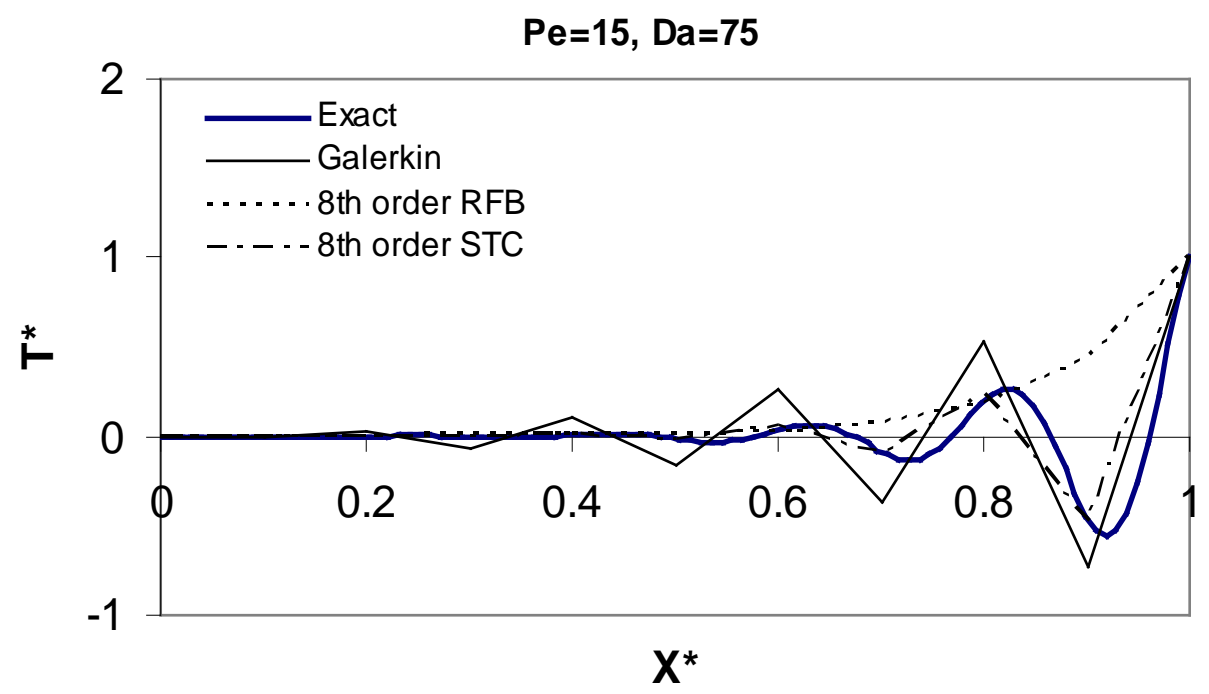

Fig. 8. Results for $P e=15$ and $D a=14,60,75$ - propagation regime 


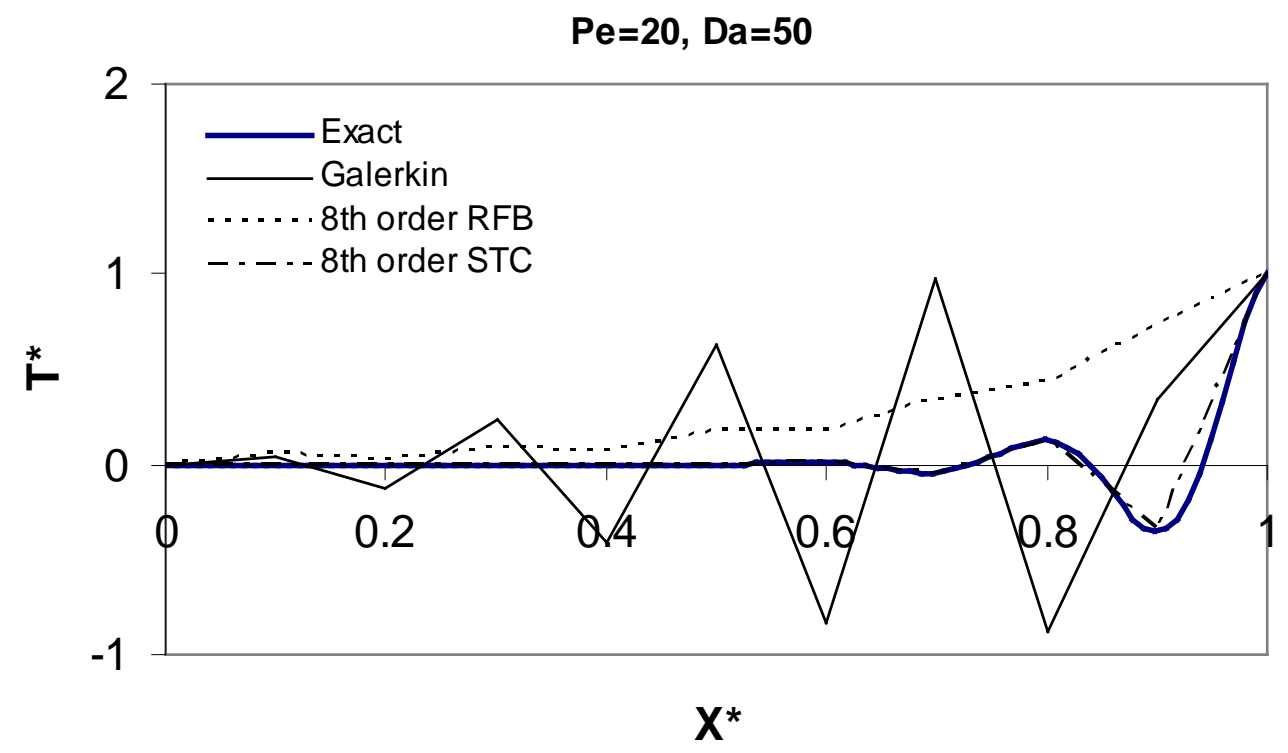

Fig. 9. Results for $P e=20$ and $D a=40,50$ - propagation regime. 